\title{
OXIDAÇÃO ELETROQUÍMICA DO HERBICIDA TEBUTIURON UTILIZANDO ELETRODO DO TIPO DSA ${ }^{\circledR}$
}

Suellen A. Alves, Tanare C. R. Ferreira e Marcos R. V. Lanza*

Departamento de Química e Física Molecular, Instituto de Química de São Carlos, Universidade de São Paulo, CP 780, 13560-970

São Carlos - SP, Brasil

Recebido em 2/3/12; aceito em 12/7/12; publicado na web em 31/8/12

ELECTROCHEMICAL OXIDATION OF THE HERBICIDE TEBUTHIURON USING DSA ${ }^{\circledR}$-TYPE ELECTRODE. Tebuthiuron $(\mathrm{TBH})$ is a herbicide widely used in different cultures and known for its toxic effects. Electrochemical methods are promising for removing pollutants such as pesticides. This study showed the degradation of TBH using a DSA ${ }^{\circledast}$ anode operated at current densities of 50 to $200 \mathrm{~mA} \mathrm{~cm}^{-2}$. Removal presented pseudo-first order kinetics while high-pressure liquid chromatography (UV detection) showed two peaks, ascribed to degradation intermediates. The maximum percentage of total organic carbon removed was $12.9 \%$. Ion chromatography revealed that higher concentrations of nitrate and nitrite ions formed with increasing current density.

Keywords: DSA-Cl ${ }_{2}^{\circledR}$; electrooxidation; tebuthiuron.

\section{INTRODUÇÃO}

O Brasil apresenta expressivo crescimento no comércio internacional e nacional do agronegócio, sendo considerado um dos maiores produtores e exportadores de alimentos. Dentro deste contexto, a cultura da cana-de-açúcar se destaca sendo o Brasil o primeiro do mundo na produção de açúcar e etanol conquistando, cada vez mais, o mercado externo com o uso do biocombustível como alternativa energética. ${ }^{1}$

Esta intensa atividade agrícola pode provocar impactos ao meio ambiente, como a contaminação dos corpos hídricos por herbicidas e pesticidas. O Tebutiuron (TBH) (Figura 1) é um herbicida amplamente utilizado na cana-de-açúcar em aplicações de pré-emergência, para controle das principais espécies anuais infestantes da cultura, sendo também aplicado no sistema de cana-crua. ${ }^{2}$<smiles>CNC(=O)N(C)c1nnc(C(C)(C)C)s1</smiles>

Figura 1. Fórmula estrutural do herbicida Tebutiuron

De acordo com a Agência de Proteção Ambiental da Califórnia, EUA, o TBH é considerado persistente e móvel, podendo contaminar o solo e os reservatórios de água. Apresenta elevada solubilidade em água e hidrólise superior a 64 dias. Seu metabolismo aeróbico no solo acorre em 1220 dias e o anaeróbico em 1520 dias. $^{3}$

Em ensaios de toxicidade, realizados utilizando três microalgas tropicais (Navicula sp. e Cylindrotheca closterium (Ochorophyta) e Nephroselms pyriformis (Chlorophyta)), o TBH está entre os pesticidas mais tóxicos, ficando atrás apenas do Diuron e da Hexazinona. ${ }^{4}$

O controle da concentração de poluentes orgânicos na água é uma importante medida de proteção ambiental. Processos eletroquímicos vêm sendo estudados como uma alternativa para o tratamento de efluentes aquosos. ${ }^{5}$ Algumas das vantagens do tratamento eletroquímico são utilização do elétron como reagente, uso do catalisador na

*e-mail: marcoslanza@iqsc.usp.br forma de revestimento de eletrodos metálicos e formação de espécies reativas na superfície do eletrodo, fornecendo uma alternativa promissora aos métodos tradicionais. ${ }^{6}$

Entre os vários eletrodos utilizados para processos eletroquímicos destacam-se os ânodos do tipo DSA (patenteado pela Diamond Shamrock Technologies S.A. em Genebra - Suiça com o nome de Dimensionally Stable Anodes, DSA ${ }^{\circledR}$ ) os quais são constituídos de um suporte metálico barato e com resistência mecânica (tal como o titânio) sobre o qual são depositadas misturas de óxidos por decomposição térmica. ${ }^{7}$ A forte adesão da mistura de óxidos ao suporte metálico é assegurada pela formação de uma camada de $\mathrm{TiO}_{2}$ a partir do Ti metálico, durante a calcinação da mistura precursora. ${ }^{7}$ Os óxidos industriais mais comuns são formados por $\mathrm{RuO}_{2}$ e $\mathrm{TiO}_{2}$ $\left(\mathrm{Ti} / \mathrm{Ru}_{0}, \mathrm{Ti}_{0}, \mathrm{O}_{2}\right)$, os quais são estáveis para a produção de $\mathrm{Cl}_{2} \mathrm{e} /$ ou processos de desprendimento de oxigênio em baixas correntes (chamados de DSA-Cl $l_{2}$ ). Ânodos contendo Ir e Ta (com ou sem a presença de $\mathrm{Sn}$ ) são estáveis em processos onde ocorre a produção de oxigênio e são conhecidos como DSA- $\mathrm{O}_{2}$. Diversos trabalhos na literatura reportam a oxidação eletroquímica de compostos como formaldeído, corantes têxteis e pesticidas utilizando eletrodos do tipo DSA com proporções distintas. ${ }^{8-10}{ }^{89}$,

Eletrodos do tipo DSA vêm sendo utilizados na eletro-oxidação de poluentes. Malpass e colaboradores estudaram a remoção do pesticida atrazina utilizando eletrodo DSA ${ }^{\circledast}$ (composição Ti/ $\mathrm{Ru}_{0},{ }_{3} \mathrm{Ti}_{0,7} \mathrm{O}_{2}$ ). Diferentes eletrólitos suportes foram utilizados para avaliar a eficiência do método e os autores relataram que a remoção de carbono orgânico total foi possível quando $\mathrm{NaCl}$ foi adicionado ao eletrólito, formando-se espécies oxidantes $\left(\mathrm{ClO}^{-}\right) .{ }^{11}$

Samet e colaboradores avaliaram a degradação eletroquímica do inseticida clorpirifós utilizando eletrodo $\mathrm{Nb} / \mathrm{PbO}_{2} \cdot{ }^{12}$ Estudaram a influência da densidade de corrente aplicada, da concentração inicial do inseticida e da temperatura durante as eletrólises. Observaram que a remoção de DQO segue cinética de pseudossegunda ordem e a eficiência na degradação é maior com o aumento da temperatura e da densidade de corrente aplicada; o aumento da concentração inicial do inseticida proporciona diminuição da remoção do mesmo. A melhor condição obtida para o tratamento eletroquímico foi com densidade de corrente de $50 \mathrm{~mA} \mathrm{~cm}^{-2}$, DQO inicial de $450 \mathrm{mg} \mathrm{O}_{2} \mathrm{~L}^{-1}$, temperatura de $70{ }^{\circ} \mathrm{C}$ com $10 \mathrm{~h}$ de eletrólise. ${ }^{12}$

A degradação dos pesticidas diuron e 3,4-dicloroanilina utilizando eletrodo de DDB foi investigada por Polcaro e colaboradores. ${ }^{13}$ Os 
pesticidas foram completamente mineralizados e não se observou a passivação do material anódico. ${ }^{13}$

No presente trabalho, estudou-se o tratamento eletroquímico do herbicida $\mathrm{TBH}$, utilizando-se eletrodo comercial $\mathrm{DSA}-\mathrm{Cl}_{2}$. Investigou-se o efeito do uso de diferentes densidade de corrente (50$200 \mathrm{~mA} \mathrm{~cm}^{-2}$ ) utilizando as técnicas analíticas espectroscópicas na região do UV-Vis, cromatografia líquida de alta eficiência (CLAE) e cromatografia de íons (IC), além do monitoramento da mineralização da matéria orgânica por análise do carbono orgânico total (COT).

\section{PARTE EXPERIMENTAL}

\section{Degradação do Tebutiuron}

O eletrodo comercial DSA-Cl ${ }_{2}^{\circledR}\left(\mathrm{Ti} / 30 \mathrm{RuO}_{2}-70 \mathrm{TiO}_{2}\right)$ utilizado foi produzido pela De Nora do Brasil Ltda. Todas as soluções foram preparadas com água purificada $(18,2 \mathrm{M} \Omega \mathrm{cm})$ obtida por um sistema de purificação Millipore Milli-Q water. As degradações eletroquímicas do herbicida TBH foram realizadas em uma célula de compartimento único, em formato cilíndrico, de polipropileno com capacidade de $450 \mathrm{~mL}$ (Figura 2).

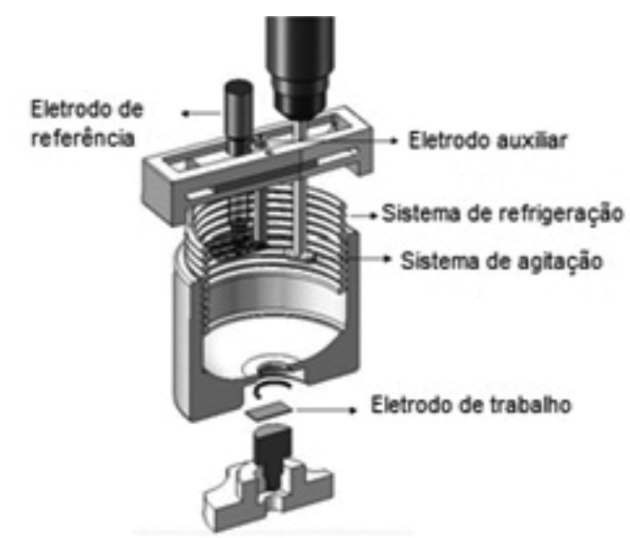

Figura 2. Representação esquemática da célula eletroquímica

O eletrodo de trabalho (aproximadamente $4,15 \mathrm{~cm}^{2}$ de área geométrica) foi posicionado no centro da base da célula. Uma tela de platina foi utilizada como contraeletrodo e como eletrodo de referência foi utilizado um eletrodo de $\mathrm{Ag} / \mathrm{AgCl}\left(\mathrm{KCl} 3 \mathrm{~mol} \mathrm{~L}^{-1}\right)$. Todos os ensaios de degradação foram realizados a $20^{\circ} \mathrm{C}$ e o sistema foi mantido sob agitação mecânica constante. A dispersão comercial (Combine $500 \mathrm{SC}^{\circledast}$ - Dow AgroSciences Ltda) foi dispersa em 0,35 L do eletrólito $0,1 \mathrm{~mol} \mathrm{~L}^{-1} \mathrm{H}_{2} \mathrm{SO}_{4}$ e $0,1 \mathrm{~mol} \mathrm{~L}^{-1} \mathrm{~K}_{2} \mathrm{SO}_{4}\left(\right.$ Ecibra $^{\circledR}$ ) com concentração de $100 \mathrm{mg} \mathrm{L}^{-1}$. As eletrólises foram realizadas em modo galvanostático em um potenciostato/galvanostato Autolab PGSTAT- 302 acoplado a um módulo de alta corrente BSRT-10A e controlado pelo software GPES EcoChimie, onde foram aplicadas as densidades de correntes de 50, 75, 100, 150 e $200 \mathrm{~mA} \mathrm{~cm}^{-2}$. Para construção das curvas de calibração no CLAE e UV-Vis utilizou-se Tebuthyuron Supelco ${ }^{\circledR}(99,9 \%$ de pureza).

\section{Instrumentação e condições de análise}

As análises da concentração de TBH foram realizadas em um cromatógrafo líquido de alta eficiência (CLAE) da Shimadzu modelo 20A com detector UV/Visível SPD-20A. A fase estacionária utilizada foi uma coluna de fase reversa (coluna $\mathrm{C}_{18}$ Varian Pursuit 5250 x 4,6 $\mathrm{mm}$ ) e como fase móvel utilizou-se água/acetonitrila (M. Tedia grau CLAE) com proporção 70:30, fluxo de $0,8 \mathrm{~mL} \mathrm{~min}^{-1}$, comprimento de onda de $249 \mathrm{~nm}$ a $40{ }^{\circ} \mathrm{C}$.
Para detecção e quantificação dos íons inorgânicos formados durante as eletrólises utilizou-se um cromatógrafo de íons (CI) modelo Pro 850 Metrohm, coluna A Supp 5 e fase móvel uma solução de $3,2 \times 10^{-3} \mathrm{~mol} \mathrm{~L}^{-1}$ de carbonato de sódio e $1,0 \times 10^{-3} \mathrm{~mol} \mathrm{~L}^{-1}$ de bicarbonato de sódio (J.T. Baker) com fluxo de $0,7 \mathrm{~mL} \mathrm{~min}^{-1}$ e detector de condutividade.

Para determinação da remoção da matéria orgânica, foi utilizado um analisador de carbono orgânico total (COT) modelo COT-VCPN Shimadzu. Os espectros UV/Vis foram registrados em um espectrofotômetro, Varian Cary 50 Scan, na faixa de 200 a 800 nm, utilizando uma cubeta de quartzo de caminho óptico de $1 \mathrm{~cm}$. A absorção máxima utilizada para o cálculo de concentração por UV/Vis foi $257 \mathrm{~nm}$.

\section{RESULTADOS E DISCUSSÃO}

Para avaliar o decaimento da concentração da solução de TBH eletrolisada, observou-se a absorbância no comprimento de onda máximo de $257 \mathrm{~nm}$, que corresponde à transição $\pi \rightarrow \pi^{*}$ do TBH com ligações duplas conjugadas. As Figuras 3 a e $3 b$ mostram os espectros UV-Vis registrados nas densidades de corrente aplicadas de 100 e 200 $\mathrm{mA} \mathrm{cm}{ }^{-2}$, respectivamente.
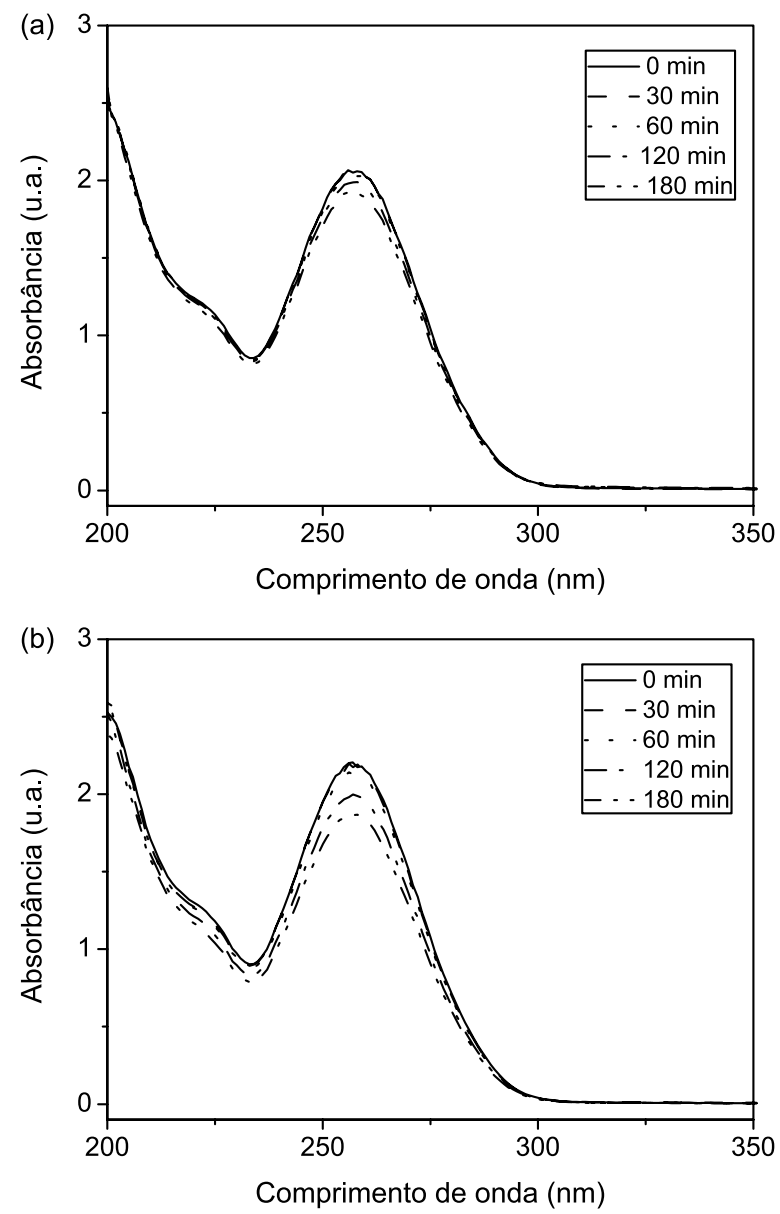

Figura 3. Espectros UV-Vis registrados durante as eletrólises de uma solução comercial de TBH nas densidades de corrente de (a) 100 e (b) $200 \mathrm{~mA} \mathrm{~cm}^{-2}$

Não foi observado aumento da absorbância acima de 400 nm, o que pode ser atribuído à ausência de intermediários de degradação que absorvem na região do visível. Na densidade de corrente de 100 $\mathrm{mA} \mathrm{cm}{ }^{-2}$, observou-se uma ligeira queda na absorbância em função do tempo de eletrólise. $\mathrm{Na}$ densidade de $200 \mathrm{~mA} \mathrm{~cm}^{-2}$, o decaimento da absorbância foi maior $(15,96 \%)$. Nas demais densidades de corrente 
estudadas ocorreu comportamento similar, entretanto na menor densidade de corrente $\left(50 \mathrm{~mA} \mathrm{~cm}^{-2}\right)$ não houve queda na absorbância.

Foram registrados cromatogramas CLAE/UV durante as eletrólises das soluções simuladas de TBH. Nas Figuras 4a, 4b e 4c são apresentados os cromatogramas da solução inicial e após 60 e 180 min de tratamento, respectivamente. Observa-se um pico no tempo de retenção de 3,40 min, o qual pode ser associado ao eletrólito. $\mathrm{Na}$ densidade de corrente de $50 \mathrm{~mA} \mathrm{~cm}{ }^{-2}$ não se observou queda na concentração do TBH, bem como o aparecimento de outros picos. A queda na área do pico e, consequentemente, na concentração do
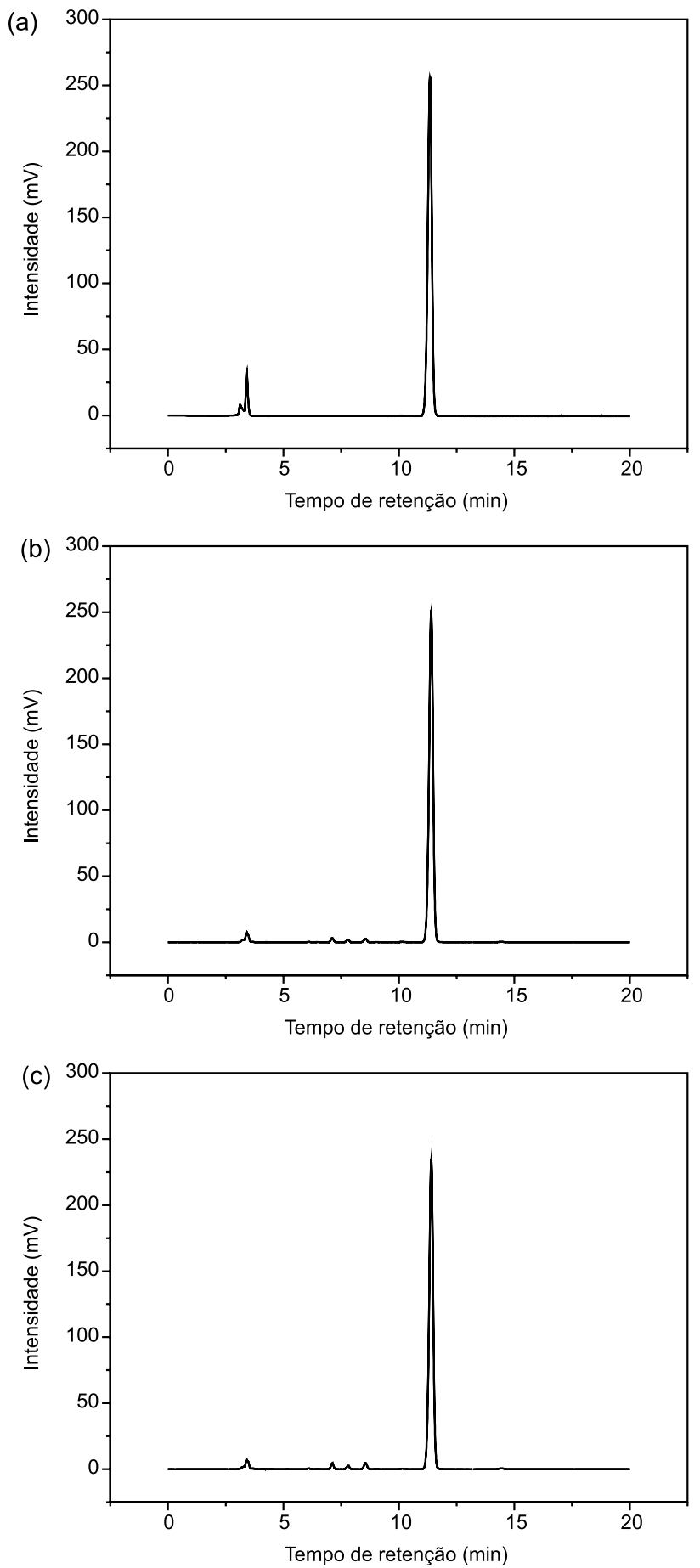

Figura 4. Cromatogramas CLAE/UV obtidos na eletrólise da solução comercial de TBH na densidade de corrente de $200 \mathrm{~mA} \mathrm{~cm}^{-2}$ (a) solução inicial, (b) $120 \operatorname{min~e}(c) 180 \mathrm{~min}$
TBH foi observada a partir de $75 \mathrm{~mA} \mathrm{~cm}^{-2}$, e três picos podem ser observados a partir de $100 \mathrm{~mA} \mathrm{~cm}^{-2}$ nos tempos de retenção de 7,08; 7,80 e 8,57 min, os quais apresentam características mais polares que o composto inicial. Tais picos podem ser atribuídos a subprodutos de degradação, uma vez que não são observados na solução inicial, e têm sua intensidade aumentada conforme o aumento do tempo de eletrólise e a densidade de corrente. A queda na concentração do TBH foi de 20,04\% na densidade de corrente de $200 \mathrm{~mA} \mathrm{~cm}^{-2}$ após $3 \mathrm{~h}$ de tratamento.

A partir de uma curva de calibração previamente construída com soluções de um padrão de TBH, obtiveram-se curvas de decaimento de concentração do TBH em função do tempo nas densidades de corrente de 75 a $200 \mathrm{~mA} \mathrm{~cm}^{-2}$ por CLAE/UV (Figura 5).

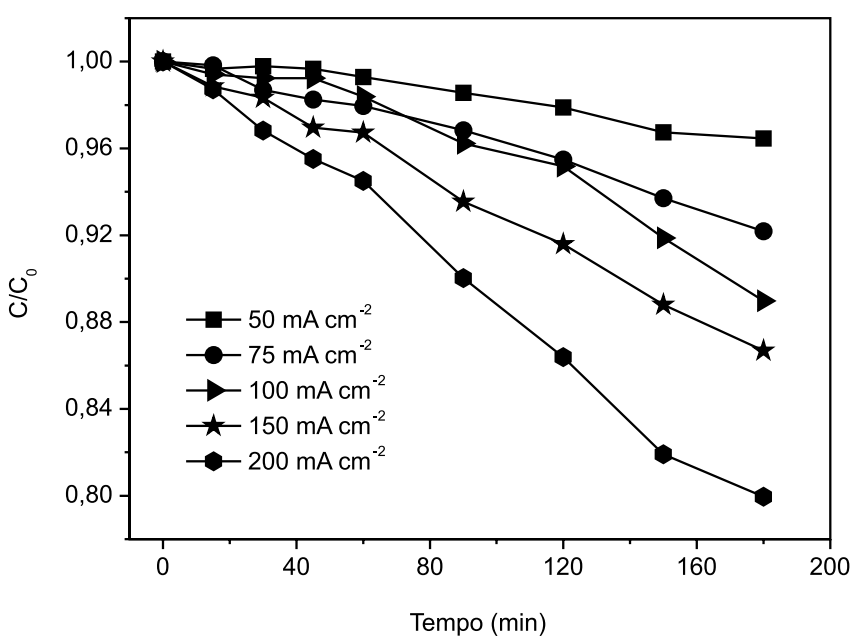

Figura 5. Curvas de decaimento da concentração de TBH em função do tempo nas densidades de corrente aplicadas de 75-200 mA cm-2 utilizando ânodo DSA e concentração inicial de 100 ppm de TBH comercial

Determinou-se a cinética da reação de remoção bem como a constante de velocidade aparente a partir da Equação 1:

$$
\ln \frac{[T B H]}{[T B H]_{\mathrm{o}}}=-k_{a p} t
$$

A cinética do processo foi considerada de pseudoprimeira ordem, devido ao comportamento linear obtido pela Equação 1, e a inclinação dessa curva foi considerada como constante de velocidade. Foram obtidos os valores de $2,16 \times 10^{-4}\left(50 \mathrm{~mA} \mathrm{~cm}^{-2}\right), 4,47 \times 10^{-4}(75 \mathrm{~mA}$ $\left.\mathrm{cm}^{-2}\right), 4,27 \times 10^{-4}\left(100 \mathrm{~mA} \mathrm{~cm}^{-2}\right), 8,04 \times 10^{-4}\left(150 \mathrm{~mA} \mathrm{~cm}^{-2}\right)$ e 1,30 x $10^{-3} \mathrm{~min}^{-1}\left(200 \mathrm{~mA} \mathrm{~cm}{ }^{-2}\right)$. Observa-se, em geral, um aumento da velocidade de remoção do TBH conforme se aumenta a densidade de corrente.

A queda na concentração não é conclusiva no que concerne à conversão a $\mathrm{CO}_{2}$. Para tanto, avaliou-se a taxa de mineralização, sendo que os valores de COT foram obtidos no início e no final de cada experimento, obtendo-se a porcentagem de matéria orgânica convertida em $\mathrm{CO}_{2}$ (Tabela 1).

É possível observar que a maior taxa de conversão foi obtida na maior densidade de corrente $\left(200 \mathrm{~mA} \mathrm{~cm}^{-2}\right)$. Nas demais densidades de corrente, a taxa de conversão foi menor, evidenciado que nessas condições se torna difícil a mineralização. Nessa densidade de corrente $20,0 \%$ da concentração da solução inicial é removida, entretanto $12,9 \%$ são convertidos $\mathrm{a} \mathrm{CO}_{2}$, fato que pode ser atribuído à formação de subprodutos de degradação.

A partir dos valores de COT, calculou-se a energia elétrica específica, a qual é um parâmetro fundamental para avaliar o processo 
Tabela 1. Porcentagem de remoção de COT e energia elétrica específica em função das densidades de corrente aplicadas

\begin{tabular}{ccc}
\hline $\begin{array}{c}\text { Densidade de corrente } \\
\left(\mathrm{mA} \mathrm{cm}^{-2}\right)\end{array}$ & Remoção de COT $(\%)$ & Es $\left(\mathrm{kWh} \mathrm{g}^{-1}\right.$ de COT $)$ \\
\hline 50 & - & - \\
75 & 6,3 & 10,7 \\
100 & 7,6 & 12,0 \\
150 & 9,0 & 21,8 \\
200 & 12,9 & 23,4 \\
\hline
\end{tabular}

de tratamento. Para calcular a energia elétrica consumida utilizou-se a Equação 2:14

$$
E c=\frac{\text { Ecel.I.t }}{1000}
$$

onde $\mathrm{E}_{\text {cel }}$ é a diferença de potencial da célula $(\mathrm{V})$, medida pela diferença dos potenciais entre o cátodo e o ânodo; I é corrente (A) e to tempo (h) e (resposta em $\mathrm{kWh}$ ). A partir da energia elétrica consumida, calculou-se energia elétrica específica através da Equação 3 (Tabela 1): ${ }^{14}$

$$
E s=\frac{\mathrm{E}_{\mathrm{c}}}{\mathrm{m}}
$$

onde $\mathrm{m}$ é a massa de COT removida em gramas. Na densidade de corrente de $50 \mathrm{~mA} \mathrm{~cm}^{-2}$ não foi possível calcular o $\mathrm{CE}$, uma vez que não houve queda no COT. O consumo energético aumentou conforme se aumenta a densidade de corrente, entretanto aumenta também a remoção total da solução eletrolisada. O sistema proposto torna-se promissor sem adição de espécies que formam oxidantes na solução eletrolisada.

Os eletrodos DSA são conhecidos como eletrodos ativos. Após a descarga de água, os radicais hidroxila gerados são adsorvidos fortemente na superfície do ânodo e formam óxidos superiores $\left(\mathrm{MO}_{\mathrm{x}+1}\right)$, conforme a Equação 4: ${ }^{15,6}$

$$
\left(\mathrm{MO}_{\mathrm{x}}\right)+\mathrm{H}_{2} \mathrm{O} \rightarrow\left(\mathrm{MO}_{\mathrm{x}+1}\right)+2 \mathrm{H}^{+}+2 \mathrm{e}^{-}
$$

Assim, $\left(\mathrm{MO}_{\mathrm{x}+1}\right)$ é a espécie ativa responsável pela oxidação da matéria orgânica e desprendimento de $\mathrm{O}_{2}$, a qual é uma reação competitiva com o processo, conforme Equações 5 e 6 :

$$
\begin{gathered}
\left(\mathrm{MO}_{\mathrm{x}+1}\right)+\mathrm{R} \rightarrow\left(\mathrm{MO}_{\mathrm{x}}\right)+\mathrm{RO} \\
\left(\mathrm{MO}_{\mathrm{x}+1}\right) \rightarrow\left(\mathrm{MO}_{\mathrm{x}}\right)+1 / 2 \mathrm{O}_{2}
\end{gathered}
$$

Essa oxidação é parcial e seletiva e a espécie $\left(\mathrm{MO}_{\mathrm{x}}\right)$ é continuamente regenerada. ${ }^{15}$ Assim, este eletrodo participa do processo de oxidação devido à formação da espécie ativa $\mathrm{MO}_{\mathrm{x}+1}$.

Observou-se a formação de íons inorgânicos durante as eletrólises, utilizando a técnica de cromatografia de íons, uma vez que nem todo o TBH degradado foi transformado em $\mathrm{CO}_{2}$, mas houve formação de subprodutos e estes podem ser íons inorgânicos, com concentrações máximas em função do tempo de eletrólise nas diferentes densidades de corrente (Tabela 2).

Verifica-se o aumento da concentração de íons nitrato em função do aumento da densidade de corrente. Conforme se aumenta a densidade de corrente, aumenta a concentração de íons até o valor máximo de 2,8 ppm desse íon. Esse íon pode ter sido formado devido ao ataque da espécie $\mathrm{MO}_{\mathrm{x}+1}$ aos átomos de $\mathrm{N}$ da molécula de TBH.

A formação de íons nitrito só pode ser observada nas densidades de corrente de 150 e $200 \mathrm{~mA} \mathrm{~cm}{ }^{-2}$, com valor máximo de $0,9 \mathrm{ppm}$. A formação desse íon pode ser atribuída à redução de íons nitrato no cátodo.
Tabela 2. Formação de íons nitrato e nitrito nas densidades de corrente aplicadas

\begin{tabular}{ccc}
\hline $\begin{array}{c}\text { Densidade de corrente } \\
\left(\mathrm{mA} \mathrm{cm}^{-2}\right)\end{array}$ & Nitrato (ppm) & Nitrito (ppm) \\
\hline 50 & 0,6 & $\mathrm{Nd}$ \\
75 & 0,8 & $\mathrm{Nd}$ \\
100 & 1,3 & $\mathrm{Nd}$ \\
150 & 1,9 & 0,5 \\
200 & 2,8 & 0,9 \\
\hline
\end{tabular}

\section{CONCLUSÕES}

O tratamento eletroquímico proposto apresentou-se promissor na remoção de uma solução simulada do herbicida tebutiuron. A queda na concentração e no carbono orgânico total só foi observada a partir da aplicação de $75 \mathrm{~mA} \mathrm{~cm}{ }^{-2}$. A cinética do processo foi considerada de pseudoprimeira ordem e a velocidade de remoção foi mais rápida na densidade de corrente de $200 \mathrm{~mA} \mathrm{~cm}^{-2}\left(1,30 \times 10^{-3} \mathrm{~min}^{-1}\right)$ e nessa condição obteve-se maior remoção de COT $(12,9 \%)$. Os cromatogramas CLAE/UV mostram o aparecimento de dois picos que podem ser associados a possíveis subprodutos de degradação. A cromatografia de íons mostra a formação de íons inorgânicos durante o processo, sendo que nitrato está relacionado à quebra da molécula e nitrito pode ser associado à redução de nitrato no cátodo.

\section{AGRADECIMENTOS}

À FAPESP e Capes pelo apoio financeiro.

\section{REFERÊNCIAS}

1. Vilar, J. P.; Pinho, X.; Pintor, A. M. A.; Boaventura, R. A. R.; Sol. Energy 2011, 85, 1927

2. Negrisoli, E. I.; Velini, E. D. I.; Rossi, C. V. S.; Correia, T. M.; Costa, A. G. F.; Planta Daninha 2007, 25, 621.

3. Dias, J.; Agriculture Cal/EPA 2011, GW08.

4. Magnusson, M.; Heimann, K.; Quayle, P.; Negri, A. P.; Mar. Pollut. Bull. 2010, 60, 1978 .

5. Alves, S. A.; Ferreira, T. C. R.; Sabatini, N. S.; Trientini, A. C. A.; Migliorini, F. L.; Baldan, M. R.; Ferreira, N. G.; Lanza, M. R. V.; Chemosphere 2012, 88,155.

6. Rajeshwar, K. Em Environmental Electrochemistry: Fundamentals and Applications in Pollution Abatement; Rajeshwar, K.; Ibanez, J., eds.; Academic Press: London, 1996, chap. 3.

7. Trasatti, S.; Technology 2000, 45, 2377.

8. Terumi, M.; Guimar, R.; Bertazzoli, R.; Chem. Eng. J. 2008, 136, 236.

9. Panakoulias, T.; Kalatzis, P.; Kalderis, D.; Katsaounis, A.; J. Appl. Electrochem. 2010, 40, 1759.

10. Aquino Neto, S.; De Andrade, A. R.; J. Appl. Electrochem. 2009, 39, 1863.

11. Malpass, G. R. P.; Miwa, D. W.; Machado, S. S.; Olivi, P.; Motheo, J.; J. Hazard. Mater. 2006, 137, 565.

12. Samet, Y.; Agengui, L.; Abdelhédi, R.; J. Electroanal. Chem. 2010 , 650,152 .

13. Polcaro, A. M.; Mascia, M.; Palmas, S.; Vacca, A.; Electrochim. Acta. 2004, 49, 649.

14. Pletcher, D. Em Industrial Electrochemistry; Pletcher, D.; Walsh, F. C., eds.; Chapman and Hall: London, 1990, chap. 2.

15. Simond, O.; Schaller, V.; Comninellis, C.; Science 2012, 42, 2009.

16. Comninellis, C.; Electrochim. Acta 1994, 39, 1857. 